\title{
INTER-RELAÇÕES ENTRE HELICOBACTER PYLORI, SENSAÇÃO SUBJETIVA DE APETITE E CONSUMO ALIMENTAR
}

\author{
Interrelationship between Helicobacter pylori infection, subjective appetite sensation and food \\ intake
}

\section{Interrelaciones entre Helicobacter pylori, sensación subjetiva del apetito y consumo de alimentos}

\author{
Laís Marinho Aguiar \\ Universidade Estadual do Ceará - UECE - Fortaleza (CE) - Brasil \\ Daianne Cristina Rocha \\ Universidade Estadual do Ceará - UECE - Fortaleza (CE) - Brasil
}

Antonio Augusto Ferreira Carioca

Universidade de São Paulo - USP - São Paulo (SP) - Brasil

Helena Alves de Carvalho Sampaio

Universidade Estadual do Ceará - UECE - Fortaleza (CE) - Brasil

\section{RESUMO}

Objetivo: Avaliar o efeito da infecção por Helicobacter pylori $(\mathrm{Hp})$ sobre a sensação subjetiva de apetite e consumo alimentar de pacientes usuários do Sistema Único de Saúde. Métodos: Trata-se de um estudo do tipo transversal, realizado em duas unidades de saúde durante o período de julho de 2012 a maio de 2013, em que foram avaliados 38 pacientes, $14 \mathrm{Hp}$ positivos e 24 negativos, que relataram fazer apenas três refeições principais por dia. A presença da infecção foi investigada a partir de endoscopia digestiva alta. O consumo alimentar foi investigado por meio de dois recordatórios de 24 horas. A sensação subjetiva de apetite foi investigada utilizando-se a escala analógica visual (EAV) de apetite. Os pacientes foram estratificados em Hp positivos e Hp negativos. Para todas as análises considerou-se significante p $<0,05$. Resultados: Não houve diferença significante no consumo de energia e de macronutrientes, por refeição, entre os grupos. A sensação subjetiva do apetite antes e após cada refeição foi semelhante entre os grupos, exceto quanto à fome $(p=0,016)$ e saciedade $(p=0,008)$ no desjejum. Constatou-se a influência da refeição anterior na refeição subsequente, sendo estes achados mais evidentes entre os pacientes Hp negativos. Conclusão: O estudo aponta homogeneidade dos dois grupos avaliados no que tange a ingestão alimentar. A sensação subjetiva do apetite é diferente entre os grupos nos tópicos fome e saciedade relacionados ao desjejum. A relação entre as sensações percebidas ao final de uma refeição e início da subsequente é mais evidente entre pacientes Hp negativos.

Descritores: Helicobacter pylori; Apetite; Dieta.

\section{ABSTRACT}

Objective: To evaluate the effect of Helicobacter pylori (Hp) infection on the subjective appetite sensation and food intake of patients using the Unified Health System. Methods: This is a cross-sectional study, carried out in two health units during the period from July 2012 to May 2013, which evaluated 14 Hp-positive and 24 Hp-negative subjects, totaling 38 patients who reported having only three main meals per day. The presence of infection was investigated through upper endoscopy. Food intake was investigated by means of two 24-hour recalls. Subjective appetite sensation was investigated with use of the Visual Analog Scale of Appetite (VAS). The patients were stratified as Hp-positive and Hpnegative. For all analyses, the significance level was $p<0.05$. Results: There was no significant difference in the energy and macronutrients intake, per meal, between the groups. The subjective appetite sensation before and after each meal was similar between groups, except for hunger $(p=0.016)$ and satiety $(p=0.008)$ at breakfast. The influence of the previous meal on the subsequent one was verified, these findings being more evident among the Hp-negative patients. Conclusion: The study showed homogeneity of the two evaluated groups in regard to food intake. The subjective appetite sensation is different between the groups on the topics hunger and satiety related to breakfast. The relationship between the sensations perceived by the end of a meal and the beginning of the subsequent one is more evident in Hp-negative.

Descriptors: Helicobacter pylori; Appetite; Diet. 


\section{RESUMEN}

Objetivo: Evaluar el efecto de la infección por Helicobacter pylori (Hp) sobre la sensación subjetiva del apetito y el consumo de alimentos de pacientes usuarios del Sistema Único de Salud. Métodos: Se trata de un estudio del tipo transversal realizado en dos unidades de salud entre julio de 2012 y mayo de 2013 en el cual se evaluaron 38 pacientes siendo 14 Hp positivos y 24 negativos que relataron comer solamente tres veces al día. La presencia de la infección ha sido investigada en la endoscopia digestiva alta. El consumo de alimentos ha sido investigado a través de dos recordatorios de 24 horas. La sensación subjetiva del apetito ha sido investigada utilizando la Escala Analógica Visual de Apetito (EAV). Los pacientes fueron clasificados en Hp positivos y Hp negativos. Para todos los análisis se consideró significativo el $p<0,05$ Resultados: No hubo diferencia significativa entre los grupos para el consumo de energía y de macro nutrientes por comida. La sensación subjetiva del apetito antes y después de cada comida ha sido parecida entre los grupos, excepto respecto el hambre (p=0,016) y la saciedad $(p=0,008)$ en ayunas. Se constató de modo más evidente entre los pacientes Hp negativos que la comida anterior influye en la siguiente.

Conclusión: El estudio sugiere la homogeneidad de los dos grupos evaluados respecto la ingesta de alimentos. La sensación subjetiva del apetito es distinta entre los grupos respecto el hambre y la saciedad en ayunas. La relación entre las sensaciones percibidas al final de cada comida y el inicio de la siguiente es más evidente entre los pacientes Hp negativos.

Descriptores: Helicobacter pylori; Apetito; Dieta.

\section{INTRODUÇÃO}

A infecção pelo Helicobacter pylori (H. pylori) é considerada um importante problema de saúde pública, pois ocorre em todo mundo e acomete mais da metade da humanidade. Concentra-se mais em países em desenvolvimento, e acomete todas as faixas etárias, atingindo $70 \%$ a $90 \%$ da população. A prevalência é menor em países desenvolvidos, situando-se entre $25 \%$ e $50 \%^{(1)}$. No Brasil, a prevalência em adultos encontra-se em torno de $82 \%{ }^{(2)}$. A melhoria das condições de vida da população, mesmo nas regiões mais subdesenvolvidas, pode promover drástica redução desse micro-organismo ${ }^{(3)}$.

A literatura, embora controversa, aponta a influência da infecção pelo micro-organismo sobre a homeostase ponderal ${ }^{(4)}$. Pode ser que o H. pylori interfira nos níveis de grelina, um peptídeo secretado principalmente no estômago, que estimula o apetite, sendo liberada na corrente sanguínea antes das refeições ${ }^{(5)}$. A hipótese é que indivíduos infectados pelo microrganismo tenham níveis de grelina diminuídos em comparação a grupos sem infecção( ${ }^{(6)}$.

Há poucos estudos que relacionam apetite, ingestão alimentar e infecção pelo H. pylori. No entanto, a influência da infecção sobre secreções digestivas, motilidade e distensão gástrica, envolvendo ou não a grelina, podem repercutir nas sensações de fome e saciedade, influenciar a ingestão alimentar e, consequentemente, o peso corporal ${ }^{(7)}$.

$\mathrm{O}$ apetite pode ser medido com o auxílio de avaliações subjetivas. Quando usadas apropriadamente, essas avaliações são reprodutíveis, sensíveis e preditíveis do consumo alimentar ${ }^{(8,9)}$. A avaliação do apetite permite uma análise mais profunda do comportamento alimentar do que a observação do simples consumo isolado do alimento ${ }^{(10)}$.

Sob o ponto de vista da prática clínica, pode ser relevante investigar o apetite de portadores e não portadores da infecção em confronto com sua ingestão calórica. Tal investigação não vem sendo realizada nas rotinas de atendimento, mas torna-se importante para possibilitar intervenção que previna perda ponderal entre portadores. Assim, o objetivo deste estudo foi avaliar o efeito da infecção por Helicobacter pylori sobre a sensação subjetiva de apetite e o consumo alimentar de pacientes usuários do Sistema Único de Saúde.

\section{MÉTODOS}

Trata-se de um estudo transversal desenvolvido em duas unidades de saúde que atendem pacientes do Sistema Único de Saúde (SUS) de Fortaleza - Ceará, sendo escolhidas unidades de ensino, pesquisa e extensão com presença de um Serviço de Endoscopia Digestiva, já que a realização do exame endoscópico é um dos métodos disponíveis para diagnóstico da presença de infecção pelo H. pylori.

A amostra, não probabilística por conveniência, foi de 144 pacientes, sendo 72 positivos para H. pylori e 72 negativos para este micro-organismo. A fim de permitir maior homogeneidade da amostra para a investigação do apetite, foram incluídos no estudo somente pacientes que realizassem apenas 3 refeições por dia, sem a presença de lanches, pois estes se mostraram muito inconstantes entre os mesmos. Assim, participaram do estudo 38 pacientes, sendo 14 positivos para H. pylori e 24 negativos para H. pylori. A coleta de dados abrangeu o período de julho de 2012 a maio de 2013.

Foram incluídos indivíduos adultos, de ambos os sexos, pacientes dos serviços citados, moradores da capital (a fim de facilitar o acompanhamento), e que realizavam apenas 3 refeições ao dia. Excluíram-se os portadores de câncer gástrico, as gestantes, os indivíduos que tivessem sido submetidos à cirurgia gástrica ou que estivessem em tratamento dietoterápico, aqueles com presença de sangramento digestivo atual e pacientes que tivessem utilizado medicação à base de inibidor de bomba de prótons e/ou antibiótico nas últimas quatro semanas. 
Obteram-se informações demográficas e socioeconômicas (sexo, idade, cor autorreferida, anos de estudo e renda mensal familiar) dos pacientes. Para realização da endoscopia digestiva alta, os pacientes compareceram em jejum absoluto de 6 horas e de 8 horas para produtos lácteos. Seguindo a rotina dos serviços, durante o exame foram colhidos, com pinça endoscópica, 3 fragmentos do antro, os quais, com auxílio de agulha descartável, foram transferidos diretamente para o tubo de ensaio contendo a solução de ureia URETEST ${ }^{\circledR}$, o qual foi guardado em ambiente próximo a $36^{\circ} \mathrm{C}$. A verificação do resultado foi realizada após 24 horas. O paciente foi considerado Helicobacter pylori positivo quando o teste da urease acusou presença do micro-organismo, que, segundo o fabricante, é demonstrada pela mudança de coloração da solução do amarelo para o vermelho. Assim, os participantes foram distribuídos em dois grupos: Helicobacter pylori positivo (Hp positivo) e Helicobacter pylori negativo (Hp negativo).

Foi realizada antropometria, aferindo-se peso e altura segundo protocolo da literatura ${ }^{(11)}$ e utilizando-se balança antropométrica, marca Filizola ${ }^{\circledR}$, com capacidade de $200 \mathrm{~kg}$, com intervalo de $100 \mathrm{~g}$ e régua antropométrica acoplada com capacidade de $2,00 \mathrm{~m}$, com intervalo de $0,5 \mathrm{~cm}$. O peso e a altura foram mensurados com o paciente descalço. Para a aferição da altura, o indivíduo permaneceu em pé e ereto, com o olhar apontando para o horizonte. Foi calculado o índice de massa corporal (IMC) $\left(\mathrm{kg} / \mathrm{m}^{2}\right)$ e o estado nutricional dos participantes foi categorizado segundo a World Health Organization (WHO) ${ }^{(12)}$, mas agrupando-se obesidade graus 1, 2 e 3 como obesidade.

Quanto à investigação da sensação subjetiva de apetite, se utilizou uma escala analógica visual (EAV) adaptada de outra já construída $^{(13)}$. Os voluntários responderam às oito questões com indagação de sensações atuais de fome, saciedade, plenitude e desejo de comer alimentos em geral ou específicos (gordurosos, salgados, doces e condimentados), conforme descrito a seguir: 1) Como está sua fome agora?; 2) Quanto saciado você se sente agora?; 3) Quanto cheio você se sente agora?; 4) Quanto você acha que pode comer agora?; 5) Você gostaria de comer algum alimento doce agora?; 6) Você gostaria de comer algum alimento salgado agora?; 7) Você gostaria de comer algum alimento temperado agora?; 8) Você gostaria de comer algum alimento gorduroso agora?

Na referida escala são exibidos valores extremos de sensação presente ou ausente, indicados em uma linha de $100 \mathrm{~mm}$ de comprimento, sem graduação. Os voluntários foram orientados a marcar uma linha vertical no ponto da escala em que achassem estar a sua sensação, antes e após cada refeição realizada. O ponto marcado pelo indivíduo foi medido com auxílio de uma régua para posterior quantificação e comparação das medidas resultantes. A avaliação dos resultados dessa escala foi realizada utilizando a pontuação marcada pelos voluntários. Utilizou-se a média das pontuações de cada questão na comparação entre os grupos. Estes procedimentos de coleta e análise de dados são descritos na literatura ${ }^{(13,14)}$.

Investigou-se o consumo alimentar por meio de dois recordatórios alimentares de 24 horas antes do exame endoscópico: um referente a um dia de consumo em domicílio, obtido por contato telefônico ou por visita domiciliar, e o outro referente ao consumo em um dia de final de semana, conforme recomendação de pesquisadores ${ }^{(15)}$. Os dados, fornecidos pelos pacientes em medidas caseiras, foram transformados em gramas ${ }^{(16)}$. Após esse procedimento, os dados foram inseridos no software DietWin Profissional $2.0^{\circledR}$ para análise de calorias e macronutrientes, estabelecendo-se o consumo diário a partir da média dos dois recordatórios realizados.

Os grupos foram comparados quanto a variáveis demográficas e socioeconômicas através do teste exato de Fisher, transformando-as em variáveis dicotômicas: idade $<30$ anos e $\geq 30$ anos; anos de estudo $\leq 8$ anos e $>8$ anos; cor autorreferida branca e não branca; renda familiar mensal $<3$ e $\geq 3$ salários mínimos; estado nutricional de eutrofia e excesso ponderal (sobrepeso e obesidade). Para comparação das médias de ingestão energética e de macronutrientes, e da pontuação nas questões da EAV de apetite, foram utilizados os testes t de Student ou Mann-Whitney para comparações independentes entre os dois grupos. Por sua vez, para comparações relacionadas, foram utilizados os testes de Wilcoxon e Friedman. Para todas as análises considerou-se significante $\mathrm{p}<0,05$.

A pesquisa foi financiada pelo Conselho Nacional de Desenvolvimento Científico e Tecnológico (CNPq) e aprovada pelo Comitê de Ética em Pesquisa com Seres Humanos da Universidade Estadual do Ceará, com número de protocolo de aprovação 11582611-4. Todos os participantes assinaram um Termo de Consentimento Livre e Esclarecido.

\section{RESULTADOS}

Entre os voluntários, $14(36,8 \%)$ eram $H$. pylori positivos e $24(63,2 \%)$ eram $H$. pylori negativos. Observa-se que a maioria era do sexo feminino, $28(73,7 \%)$, com faixa etária maior que 30 anos $(63,7 \%, \mathrm{n}=28)$, com pelo menos 9 anos de estudo (71,0\%, $\mathrm{n}=27$ ) e cor autorreferida não branca $(83,3 \%, \mathrm{n}=30)$. Em relação à renda mensal, houve maior prevalência de indivíduos com baixa renda familiar mensal $(71 \%, \mathrm{n}=27)$. Houve predomínio de excesso ponderal, ou seja, sobrepeso e obesidade $(65,8 \%$, n=25). A idade média do grupo foi 37,9 $\pm 12,3$ anos e a renda média familiar mensal foi 2,54 $\pm 2,47$ salários mínimos (Tabela I). 
Tabela I - Caracterização dos pacientes avaliados segundo dados demográficos e sócio-econômicos e presença de Helicobacter pylori. Fortaleza, Ceará, 2013.

\begin{tabular}{lcccccc}
\hline \multirow{2}{*}{ Variáveis } & \multicolumn{2}{c}{ Hp negativos } & \multicolumn{2}{c}{ Hp positivos } & \multicolumn{2}{c}{ Total } \\
\cline { 2 - 7 } & $\mathbf{n}$ & $\mathbf{\%}$ & $\mathbf{n}$ & $\mathbf{\%}$ & $\mathbf{n}$ & $\mathbf{\%}$ \\
\hline Sexo & 5 & 20,8 & 5 & 35,7 & 10 & 26,3 \\
$\quad$ Masculino & 19 & 79,2 & 9 & 64,3 & 28 & 73,7 \\
$\quad$ Feminino & & & & & & \\
$\quad$ Faixa etária & 5 & 20,8 & 5 & 35,7 & 10 & 26,3 \\
$\quad<30$ & 19 & 79,2 & 9 & 64,3 & 28 & 63,7 \\
$\quad \geq 30$ & & & & & & \\
$\quad$ Anos de estudo & 5 & 20,8 & 6 & 42,9 & 11 & 29,0 \\
$\quad \leq 8$ & 19 & 79,2 & 8 & 57,1 & 27 & 71,0 \\
$\quad>8$ & & & & & & \\
$\quad$ Cor (autorreferida) & 4 & 18,2 & 2 & 14,3 & 6 & 16,7 \\
$\quad$ Branca & 18 & 81,7 & 12 & 85,7 & 30 & 83,3 \\
$\quad$ Não branca & 18 & 75,0 & 9 & 64,3 & 27 & 71,0 \\
$\quad$ Renda familiar mensal & 6 & 24,9 & 5 & 35,6 & 11 & 28,9 \\
$\quad<3$ & & & & & & \\
$\quad \geq 3$ & 8 & 33,3 & 5 & 35,7 & 13 & 34,2 \\
$\quad$ Estado nutricional & 16 & 66,6 & 9 & 64,3 & 25 & 65,8 \\
$\quad$ Eutrofia & & & & & & \\
$\quad$ Excesso ponderal & &
\end{tabular}

Hp: Helicobacter pylori

Não houve diferença entre os grupos (Hp positivo e negativo) na ingestão de energia e macronutrientes (proteínas, carboidratos e lipídeos) (Tabela II). A escala subjetiva de apetite, os valores médios obtidos para cada questão e o antes e após cada refeição, segundo o grupo dos participantes, foram expressos na tabela III. Observa-se que há homogeneidade dos grupos, exceto quanto às questões $1 \mathrm{e} 2$ no desjejum.

Tabela II - Média de ingestão dietética de calorias, proteínas, carboidratos e lipídeos, por refeição, em pacientes Hp positivo e Hp negativo acompanhados pelo Sistema Único de Saúde. Fortaleza, Ceará, 2013.

\begin{tabular}{llccc}
\hline Refeições & & Hp positivo & Hp negativo & p-valor* \\
\hline \multirow{3}{*}{ Desjejum } & Energia, kcal & $270,7(126,5)$ & $341,7(181,8)$ & 0,206 \\
& Proteínas, g & $10,0(5,4)$ & $13,6(15,4)$ & 0,408 \\
& Carboidratos, g & $35,5(16,1)$ & $51,9(35,2)$ & 0,110 \\
& Lipídios, g & $10,1(6,1)$ & $11,8(9,5)$ & 0,544 \\
Almoço & Energia, kcal & $698,5(342,6)$ & $640,8(243,6)$ & 0,548 \\
& Proteínas, g & $32,4(17,0)$ & $39,4(19,1)$ & 0,260 \\
& Carboidratos, g & $78,0(45,9)$ & $55,4(40,2)$ & 0,336 \\
\multirow{3}{*}{ Jantar } & Lipídios, g & $27,7(12,1)$ & $26,1(11,1)$ & 0,694 \\
& Energia, kcal & $447,1(236,1)$ & $516,4(245,0)$ & 0,399 \\
& Proteínas, g & $17,7(10,4)$ & $24,2(14,7)$ & 0,152 \\
& Carboidratos, g & $55,4(40,2)$ & $56,4(32,8)$ & 0,932 \\
\hline
\end{tabular}

*A comparação das médias deu-se pelo teste t de Student. Considerou-se significante $\mathrm{p}<0,05$. Hp: Helicobacter pylori. g: grama; kcal: quilocaloria. 
Tabela III - Média de valores obtidos na escala subjetiva de apetite antes e após as refeições em pacientes Hp positivo e Hp negativo acompanhados pelo Sistema Único de Saúde. Fortaleza, Ceará, 2013.

\begin{tabular}{|c|c|c|c|c|c|c|c|c|}
\hline \multirow{2}{*}{ Questão } & & \multicolumn{3}{|c|}{ Hp positivo } & \multicolumn{4}{|c|}{ Hp negativo } \\
\hline & & Antes & Depois & Diferença $^{a}$ & Antes & Depois & Diferença $^{a}$ & p-valor* \\
\hline \multirow{8}{*}{ Desjejum } & 1 & 4,6 & 3,1 & $-1,4(3,0)$ & 5,9 & 1,6 & $-4,3(4,3)$ & 0,016 \\
\hline & 2 & $5,5^{\#}$ & 6,4 & $1,0(2,7)$ & $2,9^{\#}$ & 7,1 & $4,2(4,3)$ & 0,008 \\
\hline & 3 & $6,0^{\#}$ & 7,5 & $1,5(2,5)$ & $3,5^{\#}$ & 6,7 & $3,2(4,4)$ & 0,345 \\
\hline & 4 & 5,1 & $3,9^{\#}$ & $-1,2(2,8)$ & 4,4 & $1,8^{\#}$ & $-2,6(4,0)$ & 0,201 \\
\hline & 5 & 7,2 & 7,5 & $0,3(4,7)$ & 7,7 & 8,0 & $0,3(2,4)$ & 0,273 \\
\hline & 6 & 5,9 & 7,5 & $2,0(3,8)$ & 5,2 & 8,6 & $3,4(4,5)$ & 0,410 \\
\hline & 7 & 7,1 & 7,3 & $0,3(2,5)$ & 7,4 & 7,9 & $0,5(2,1)$ & 0,964 \\
\hline & 8 & 7,7 & 8,4 & $0,7(2,0)$ & 7,6 & 9,1 & $1,5(3,5)$ & 0,777 \\
\hline \multirow{8}{*}{ Almoço } & 1 & 4,4 & 2,7 & $-1,7(3,5)$ & 5,2 & 2,1 & $-3,1(5,3)$ & 0,135 \\
\hline & 2 & 3,8 & 7,1 & $3,3(3,4)$ & 3,4 & 7,8 & $4,3(3,8)$ & 0,445 \\
\hline & 3 & 5,1 & 7,3 & $2,2(3,2)$ & 3,2 & 8,1 & $4,9(4,2)$ & 0,082 \\
\hline & 4 & 5,6 & 2,7 & $-2,9(3,6)$ & 5,2 & 1,4 & $-3,9(3,6)$ & 0,361 \\
\hline & 5 & 8,7 & $8,6^{\#}$ & $-0,1(3,4)$ & 7,6 & $5,0^{\#}$ & $-2,6(5,1)$ & 0,071 \\
\hline & 6 & 6,2 & 8,1 & $1,9(4,0)$ & 6,5 & 9,0 & $2,4(4,6)$ & 0,560 \\
\hline & 7 & 4,8 & 8,9 & $4,1(4,1)$ & 4,1 & 7,9 & $3,9(5,8)$ & 1,000 \\
\hline & 8 & 7,1 & 9,4 & $2,3(3,1)$ & 7,9 & 9,1 & $1,2(2,6)$ & 0,501 \\
\hline \multirow{8}{*}{ Jantar } & 1 & 4,5 & 4,1 & $-0,6(3,2)$ & 5,0 & 3,4 & $-1,4(5,7)$ & 0,463 \\
\hline & 2 & 5,1 & 6,5 & $0,8(4,0)$ & 3,1 & 6,5 & $3,2(5,8)$ & 0,260 \\
\hline & 3 & 5,2 & 7,1 & $1,3(3,8)$ & 3,8 & 7,6 & $3,5(5,4)$ & 0,223 \\
\hline & 4 & 5,1 & 3,0 & $-2,2(3,3)$ & 4,3 & 2,0 & $-2,1(4,7)$ & 0,709 \\
\hline & 5 & 7,9 & 6,3 & $-2,0(3,5)$ & 6,9 & 5,7 & $-1,2(5,5)$ & 0,893 \\
\hline & 6 & 5,9 & 9,1 & $2,3(3,7)$ & 6,1 & 8,1 & $1,8(4,9)$ & 0,687 \\
\hline & 7 & 6,5 & 8,0 & $0,8(4,8)$ & 6,0 & 8,2 & $2,0(4,9)$ & 0,823 \\
\hline & 8 & 7,9 & 8,7 & $0,1(2,8)$ & 8,7 & 8,8 & $0,1(3,3)$ & 0,709 \\
\hline
\end{tabular}

aValores expressos em média e desvio padrão.** A comparação das médias deu-se pelo teste Mann-Whitney." Comparação das médias do antes e depois entre os grupos. Considerou-se significante $\mathrm{p}<0,05$.

Questão 1: fome; Questão 2: saciedade; Questão 3: plenitude; Questão 4: desejo de comer; Questão 5: desejo de comer alimentos doces; Questão 6: desejo de comer alimentos salgados; Questão 7: desejo de comer alimentos temperados; Questão 8: desejo de comer alimentos gordurosos.

Hp: Helicobacter pylori.

Em relação à questão 1, que mede a sensação de fome, os pacientes Hp negativos apresentaram valores mais altos antes do café da manhã e menores após esta refeição $(\mathrm{p}=0,016)$. Já em relação à questão 2 , que mede a sensação de saciedade, os indivíduos Hp negativos referiram menos saciedade antes do desjejum em comparação com o grupo Hp positivo ( $\mathrm{p}<0,05)$, mas aumento significativo da saciedade após o desjejum só foi observado no grupo Hp negativo ( $\mathrm{p}=0,008)$.

A Tabela IV exibe os achados relativos à análise da sensação subjetiva de apetite antes e após cada refeição, segundo cada grupo avaliado. Observa-se que as sensações pré e pós-refeição são semelhantes para cada refeição em ambos os grupos, mas algumas diferenças se destacaram. No caso dos pacientes Hp positivos, a saciedade antes do almoço é menor do que a percebida antes do desjejum e do jantar ( $\mathrm{p}=0,028)$. Já entre os pacientes negativos, as diferenças ocorreram depois das refeições: a redução da fome e da saciedade é menor após o jantar e o desejo por alimento doce é menor após o desjejum $(\mathrm{p}<0,05)$. 
Tabela IV - Análise da sensação subjetiva de apetite antes e após cada refeição de pacientes Hp positivo e Hp negativo acompanhados pelo Sistema Único de Saúde. Fortaleza, Ceará, 2013.

\begin{tabular}{lcccccccc}
\hline Hp positivo & Antes & \multicolumn{7}{c}{ Depois } \\
\hline EAV & Desjejum & Almoço & Jantar & $\mathbf{p}^{*}$ & Desjejum & Almoço & Jantar & p-valor** \\
1 & 4,6 & 4,4 & 4,5 & 0,663 & 3,1 & 2,7 & 4,1 & 0,273 \\
2 & $5,5^{\mathrm{a}}$ & $3,8^{\mathrm{b}}$ & $5,1^{\mathrm{a}}$ & $\mathbf{0 , 0 2 8}$ & 6,4 & 7,1 & 6,5 & 0,132 \\
3 & 6,0 & 5,1 & 5,2 & 0,178 & 7,5 & 7,3 & 7,1 & 0,832 \\
4 & 5,1 & 5,6 & 5,1 & 0,920 & 3,9 & 2,7 & 3,0 & 0,852 \\
5 & 7,2 & 8,7 & 7,9 & 0,431 & 7,5 & 8,6 & 6,3 & 0,670 \\
6 & 5,9 & 6,2 & 5,9 & 0,898 & 7,5 & 8,1 & 9,1 & 0,472 \\
7 & 7,1 & 4,8 & 6,5 & 0,053 & 7,3 & 8,9 & 8,0 & 0,761 \\
8 & 7,7 & 7,1 & 7,9 & 0,853 & 8,4 & 9,4 & 8,7 & 0,900 \\
\hline Hp negativo & Antes & & & & Depois & & & \\
\hline EAV & Desjejum & Almoço & Jantar & $\mathbf{p}^{*}$ & Desjejum & Almoço & Jantar & p-valor* \\
1 & 5,9 & 5,2 & 5,0 & 0,906 & $1,6^{\mathrm{a}}$ & $2,1^{\mathrm{a}}$ & $3,4^{\mathrm{b}}$ & $\mathbf{0 , 0 4 2}$ \\
2 & 2,9 & 3,4 & 3,1 & 0,696 & $7,1^{\mathrm{a}}$ & $7,8^{\mathrm{a}}$ & $6,5^{\mathrm{b}}$ & $\mathbf{0 , 0 0 9}$ \\
3 & 3,5 & 3,2 & 3,8 & 0,331 & 6,7 & 8,1 & 7,6 & 0,291 \\
4 & 4,4 & 5,2 & 4,3 & 0,250 & 1,8 & 1,4 & 2,0 & 0,504 \\
5 & 7,7 & 7,6 & 6,9 & 0,516 & $8,0^{\mathrm{a}}$ & $5,0^{\mathrm{b}}$ & $5,7^{\mathrm{b}}$ & $\mathbf{0 , 0 3 6}$ \\
6 & 5,2 & 6,5 & 6,1 & 0,888 & 8,6 & 9,0 & 8,1 & 0,133 \\
7 & 7,4 & 4,1 & 6,0 & 0,069 & 7,9 & 7,9 & 8,2 & 0,886 \\
8 & 7,6 & 7,9 & 8,7 & 0,557 & 9,1 & 9,1 & 8,8 & 0,098 \\
\hline
\end{tabular}

*Teste Friedman. Letras diferentes simbolizam diferença estatística.

Questão 1: fome; Questão 2: saciedade; Questão 3: plenitude; Questão 4: desejo de comer; Questão 5: desejo de comer alimentos doces; Questão 6: desejo de comer alimentos salgados; Questão 7: desejo de comer alimentos temperados; Questão 8: desejo de comer alimentos gordurosos.

Hp: Helicobacter pylori. EAV: Escala Analógica Visual

Foi avaliado o impacto da sensação subjetiva do apetite após uma refeição sobre as sensações percebidas antes da refeição subsequente. Assim, foram comparados os valores da EAV de apetite após o desjejum com os valores antes do almoço e, da mesma forma, os valores após o almoço com os valores antes do jantar segundo cada grupo de participantes (Tabela V).

Tabela V - Influência da refeição anterior na refeição seguinte de pacientes Hp positivos e negativos acompanhados pelo Sistema Único de Saúde. Fortaleza, Ceará, 2013.

\begin{tabular}{lcccccc}
\hline $\begin{array}{l}\text { Hp positivo } \\
\text { Questão }\end{array}$ & Depois desjejum & Antes almoço & p-valor & Depois almoço & Antes jantar & p-valor \\
\hline 1 & 3,1 & 4,4 & 0,347 & 2,7 & 4,5 & 0,181 \\
2 & 6,4 & 3,8 & $\mathbf{0 , 0 0 4}$ & 7,1 & 5,1 & $\mathbf{0 , 0 1 3}$ \\
3 & 7,5 & 5,1 & $\mathbf{0 , 0 0 5}$ & 7,3 & 5,2 & 0,026 \\
4 & 3,9 & 5,6 & 0,109 & 2,7 & 5,1 & $\mathbf{0 , 0 3 3}$ \\
5 & 7,5 & 8,7 & 0,790 & 8,6 & 7,9 & 0,505 \\
6 & 7,5 & 6,2 & 0,050 & 8,1 & 5,9 & $\mathbf{0 , 0 2 1}$ \\
7 & 7,3 & 4,8 & $\mathbf{0 , 0 1 6}$ & 8,9 & 6,5 & $\mathbf{0 , 0 2 8}$ \\
8 & 8,4 & 7,1 & 0,161 & 9,4 & 7,9 & $\mathbf{0 , 0 3 8}$ \\
\hline Hp negativo & Depois desjejum & Antes almoço & $\mathbf{p}$-valor & Depois almoço & Antes jantar & p-valor \\
Questão & 1,6 & 5,2 & $\mathbf{0 , 0 0 2}$ & 2,1 & 5,0 & $\mathbf{0 , 0 0 9}$ \\
\hline 1 & 7,1 & 3,4 & $\mathbf{0 , 0 0 3}$ & 7,8 & 3,1 & $<\mathbf{0 , 0 0 1}$ \\
2 & 6,7 & 3,2 & $<\mathbf{0 , 0 0 1}$ & 8,1 & 3,8 & $\mathbf{0 , 0 0 1}$ \\
3 & 1,8 & 5,2 & $\mathbf{0 , 0 0 2}$ & 1,4 & 4,3 & $\mathbf{0 , 0 0 2}$ \\
4 & 8,0 & 7,6 & 0,861 & 5,0 & 6,9 & 0,088 \\
5 & 8,6 & 6,5 & $\mathbf{0 , 0 7 4}$ & 9,0 & 6,1 & $\mathbf{0 , 0 0 9}$ \\
6 & 7,9 & 4,1 & $\mathbf{0 , 0 3 1}$ & 7,9 & 6,0 & 0,198 \\
7 & 9,1 & 7,9 & $\mathbf{0 , 0 2 3}$ & 9,1 & 8,7 & 0,109 \\
8 & & & & & & \\
\hline
\end{tabular}

*Teste Wilcoxon. Questão 1: fome; Questão 2: saciedade; Questão 3: plenitude; Questão 4: desejo de comer; Questão 5: desejo de comer alimentos doces; Questão 6: desejo de comer alimentos salgados; Questão 7: desejo de comer alimentos temperados; Questão 8: desejo de comer alimentos gordurosos. Hp: Helicobacter pylori. 
Entre os pacientes Hp positivos, uma maior média de saciedade e plenitude depois do desjejum foi associada a uma menor saciedade $(\mathrm{p}=0,004)$ e plenitude $(\mathrm{p}=0,005)$ antes do almoço; e maior saciedade $(\mathrm{p}=0,013)$ e plenitude $(\mathrm{p}=0,026)$ após o almoço também se relacionaram a uma menor saciedade e plenitude antes do jantar. Uma maior média de desejo por alimentos salgados após o almoço impactou em um menor desejo por eles antes do jantar $(\mathrm{p}=0,021)$. Perfil semelhante foi observado no desejo por alimentos temperados após o café da manhã e antes do almoço $(\mathrm{p}=0,016)$. Corroborando com esses achados, resultados semelhantes foram descritos nas questões sobre alimentos temperados e gordurosos depois almoço, e com impacto imediato antes do jantar.

Considerando os pacientes $\mathrm{Hp}$ negativos, mais diferenças estatísticas puderam ser observadas, sempre ocorrendo que baixa fome, alta saciedade e plenitude, e baixo desejo alimentar, após o desjejum e após o almoço, se associaram a sensações contrárias antes da refeição subsequente. No caso de desejo por alimentos específicos, o menor desejo por alimentos salgados, temperados e gordurosos após o desjejum se associou a maior desejo por eles antes do almoço. O menor desejo por alimentos salgados após o almoço se associou a um maior desejo por eles antes do jantar (Tabela V).

\section{DISCUSSÃO}

O perfil demográfico e socioeconômico encontrado entre os participantes do presente estudo aponta semelhanças entre os portadores e não portadores da infecção por $H$. pylori, o que elimina potenciais fatores de confusão na interpretação dos achados relativos ao consumo alimentar e sensação subjetiva do apetite.

$\mathrm{Na}$ inclusão do estudo, pretendia-se avaliar um número equitativo de pessoas em cada grupo. Entretanto, a necessidade de se padronizar o número de refeições diárias (três refeições principais em ambos os grupos) para uma análise mais acurada da interpretação da sensação subjetiva de apetite resultou por incluir maioria de não portadores. Esse rigor para melhor interpretação dos dados, essa seleção pelas refeições realizadas, pode ser considerado um viés da pesquisa.

A classificação do estado nutricional mais prevalente (55,3\%), tanto em positivos como em negativos, foi a de sobrepeso. Esse dado é consistente com um levantamento realizado pelo IBGE através da POF 2008/2009(16), que apontou 49\% de excesso de peso na população brasileira acima de 20 anos de idade, e pela pesquisa realizada pelo Vigitel ${ }^{(17)}$, que relatou uma predominância de indivíduos com excesso de peso $(55,7 \%)$, independente do sexo, na população residente em Fortaleza.

No entanto, a literatura específica a respeito da associação de estado nutricional e condição de portador de $H$. pylori é controversa. Há estudo em que há uma associação entre os indicadores ${ }^{(18)}$, havendo maior prevalência de excesso de peso entre portadores da infecção. Outros autores não identificam essa associação ${ }^{(19,20)}$ ou constatam, ao contrário, que portadores são mais magros que não portadores ${ }^{(21)}$. Em estudo realizado com pacientes erradicados ${ }^{(22)}$ percebeu-se que não houve diferenças estatísticas em relação ao estado nutricional antes e após tratamento de erradicação.

Quando se compara o consumo de energia e macronutrientes nas três refeições percebe-se que não houve diferença significativa entre os dois grupos avaliados na atual pesquisa, evidenciando que a presença do micro-organismo não influenciou a composição da dieta.

A presença de $H$. pylori não afetou a ingestão de alimentos em relação à composição química da dieta, e, além disso, não houve associação considerando o sexo dos pacientes ${ }^{(7)}$. Contudo, em outro estudo ${ }^{(23)}$ realizado com portadores e não portadores de $H$. pylori houve uma menor ingestão de gordura por crianças Hp positivas, porém sem diferença significativa.

Um estudo ${ }^{(24)}$ que focalizou pacientes erradicados, apontou que a erradicação do microorganismo também não influenciou a ingestão alimentar e, em relação ao estado nutricional, foi observado que uma maior proporção de excesso ponderal (préobesidade e obesidade) ocorreu entre participantes erradicados quando comparados os pacientes positivos e negativos.

Apesar do conhecimento de que não se ingere alimentos apenas quando se está com fome e de que não se interrompe uma refeição apenas quando se está saciado ${ }^{(25)}$, os dados sobre as sensações subjetivas de apetite aqui encontrados mostram alguns pontos que merecem aprofundamento futuro.

Curiosamente no presente estudo, a questão 1 , referente à fome no desjejum, diferente do esperado, foi respondida em seu nível intermediário por ambos os grupos e para todas as refeições, evidenciando que os pacientes não estão com muita fome na hora da refeição. Para as questões 2, 3, que aferem, respectivamente, saciedade e plenitude esperava-se valores mais baixos antes e mais elevados após a refeição. Por sua vez, para a questão 4, que afere plenitude, esperava-se valores mais altos antes e mais baixos após a refeição.

Houve homogeneidade entre os grupos, exceto quando referente às questões 1 e 2 , sobre fome e saciedade no desjejum. Os pacientes Hp negativos apresentaram maiores médias de fome e saciedade antes do desjejum, mas redução dessa média depois da refeição. Já os Hp positivos, apresentaram menor eliminação de fome depois da refeição. Quanto à saciedade, os Hp positivo referiram menos saciedade.

Em relação à escala de apetite aplicada em outro estudo(7), houve uma homogeneidade da distribuição dos valores nos dois grupos, o que não coincide com relatos de que as respostas não são iguais, havendo modulações fisiológicas e psicológicas do apetite em grupos específicos de indivíduos, embora experiências comuns possam sincronizar perfis de fome, plenitude e outras sensações ${ }^{(26)}$. Embora não tenha havido diferenças na composição energética e de macronutrientes, pode ter havido ingestão diferenciada quanto ao tipo de alimento, aspecto que não foi aqui investigado. De fato, tem sido relatado que as qualidades sensoriais dos alimentos, entre outros fatores, contribuem para a modulação da saciedade ${ }^{(14)}$. 
No presente estudo, o grupo Hp positivo apresentou um maior desejo por doces após o almoço e o jantar. Os estudos analisando esse quesito são escassos, mas uma outra pesquisa ${ }^{(7)}$ também detectou maior desejo por alimentos doces após o almoço e o jantar, embora sem diferença significativa quanto à presença da bactéria $H$. pylori. A exemplo do encontrado por esses autores, também no presente estudo não se evidenciou desejo por alimentos gordurosos nem antes nem após as três refeições, em nenhum dos dois grupos. Pode-se especular que a palavra "gorduroso" desencadeia um juízo de que é inadequado, inibindo a marcação na escala.

Considerando sensações de apetite pré e pós-refeição, segundo a presença ou não da infecção, percebeu-se que os pacientes que possuem a bactéria apresentaram maior saciedade após o desjejum e o almoço, e menor saciedade antes do almoço $(\mathrm{p}=0,004)$ e do jantar ( $\mathrm{p}=0,013)$, fato que ocorre para a população em geral em outras pesquisas ${ }^{(27)}$.

Em estudo realizado com crianças ${ }^{(4)}$ foi observado que aquelas que possuíam H. pylori apresentaram um aumento nos níveis de leptina e de gastrina, assim como uma redução nos níveis de grelina plasmática. Esse fenômeno, portanto, pode contribuir para alterações no apetite.

Os resultados foram bem significativos ao se analisar a influência de uma refeição sobre a seguinte. Os dados deste estudo mostram que houve significância nas respostas às perguntas referentes à saciedade e ao desejo por alimentos específicos (temperado, salgado e gorduroso) em pacientes positivos, e nas perguntas referentes à fome, saciedade e predileção por alimentos salgados em pacientes negativos. Percebeu-se também que, em Hp negativos, o menor desejo por alimentos salgados, temperados e gordurosos se associou com um maior desejo por esses alimentos antes do almoço, e, ao mesmo tempo, o menor desejo por salgados após o almoço se associou com maior desejo antes do jantar. Já nos pacientes positivos, o baixo desejo por alimentos após o almoço resultou em um maior desejo antes do jantar, e, ao mesmo tempo, o baixo desejo por temperados após o desjejum impactou em um maior desejo desses alimentos antes do almoço.

O estudo apresenta algumas limitações, destacando-se o tamanho pequeno da amostra e a possibilidade de preenchimento inadequado ou incompleto da escala, já que era preenchida várias vezes ao dia.

Ressalta-se que há poucos estudos que relacionam apetite, ingestão alimentar e infecção por $H$. pylori. Tem-se a necessidade, portanto, de mais estudos para que confirmem se realmente há e, caso existam, quais os tipos de associações existentes entre essa infecção, o apetite, a ingestão alimentar e o estado nutricional, bem como quais os fatores envolvidos em tais associações. Os achados do presente estudo constituem um ponto de partida para novas investigações e, principalmente, apontam para a necessidade de se avaliar a sensação subjetiva do apetite versus consumo alimentar na prática clínica junto aos pacientes portadores e não portadores de $H$. pylori, viabilizando um atendimento mais amplo e aprofundado.

\section{CONCLUSÃO}

O estudo apontou homogeneidade dos dois grupos avaliados no que tange à ingestão alimentar. A sensação subjetiva do apetite é diferente entre os grupos nos tópicos fome e saciedade quando relacionados ao desjejum. A relação entre as sensações percebidas ao final de uma refeição e início da subsequente foi mais evidente entre pacientes Hp negativos.

\section{REFERÊNCIAS}

1. Rosa FB. A infecção por Helicobacter pylori em populações de Angola [tese] Lisboa: Universidade Nova de Lisboa: Faculdade de Ciências Médicas; 2015.

2. World Gastroenterology Organization - WGO. Practice Guidelines: helicobacter pylori nos países em desenvolvimento. Wisconsin: WGO; 2010.

3. Pontes RML. A erradicação do helicobacter pylori na atualidade e o problema da resistência [dissertação] Porto: Universidade do Porto; 2014.

4. Franceschi F, Annalisa T, Teresa DR, Giovanna D, Ianiro G, Franco S, et al. Role of helicobacter pylori infection on nutrition and metabolism. World J Gastroenterol. 2014;20(36):12809-17.

5. Serrenho DVL. Ghrelin receptor activation regulates hippocampal spine dynamics. [dissertação] Coimbra: Universidade de Coimbra; 2016.

6. Polinska B, Matowicka JK, Kemona H. The role of ghrelin in the organism. Postepy Hig Med Dosw (online). 2016;65:1-7.

7. Rocha DC, Sampaio HAC, Lima JWO, Carioca AAF, Lima GP, Oliveira TR, et al. Association of Helicobacter pylori infection with nutritional status and food intake. Nutr Hosp. 2015;32(2):905-12.

8. Ribeiro G, Santos O, Camacho M, Torres S, Mucha-Vieira F, Sampaio D, et al. Translation, Cultural Adaptation and Validation of the Power of Food Scale for Use by Adult Populations in Portugal. Act Med Port. 2015;28(5):575-82.

9. Daundasekara SS, Beasley AD, O'Connor DP, Sampson M, Hernadez D, Ledoux T. Validation of the intuitive Eating Scale for pregnant women. Appetite. 2017;112:201-9. 
10. Stubbs RJ, Hughes DA, Johnstone AM, Rowley E, Reid C, Elia M, et al. The use of visual analogue scales to assess motivation to eat in human subjects: a review of their reliability and validity with an evaluation of new hand-held computerized systems for temporal tracking of appetite ratings. Br J Nutr. 2000;84(4):405-15.

11. Alvarez BR, Pavan AL. Alturas e comprimentos. In: Petroski EL, editor. Antropometria: técnicas e padronizações. Porto Alegre: Pallotti; 1999. p. 29-51.

12. World Health Organization. Obesity preventing and managing the global epidemic. Geneva: WHO; 1998.

13. Flint A, Raben A, Blundell JE, Astrup A. Reproducibility, power and validity of visual analogue scales in assessment of appetite sensations in single test meal studies. Int J Obes Relat Metab Disord. 2000;24(1):38-48.

14. Lluch A, Hanet-Geisen N, Salah S, Salas-Salvado S, Bouron DL, Halford JCG. Short-term appetite-reducing effects of a low-fat dairy product enriched with protein and fibre. Food Qual Prefer.2010;21(4):402-9.

15. Fisberg RM, Marchioni DML, Colucci ACA. Avaliação do consumo alimentar e da ingestão de nutrientes na prática clínica. Arq Bras Endocrinol Metab. 2009;53(5):617-24.

16. Instituto Brasileiro de Geografia e Estatística. Pesquisa de orçamentos familiares 2008-2009: antropometria e estado nutricional de crianças, adolescentes e adultos no Brasil. Rio de Janeiro: IBGE; 2010.

17. Ministério da Saúde (BR), Secretaria de Vigilância em Saúde. Vigilância de fatores de risco e proteção para doenças crônicas por inquérito telefônico: VIGITEL 2014. Brasília; 2015.

18. Kopacova M, Koupil I, Seifert B, Fendrichova MS, Spirkova J, Vorisek V, et al. Body indices and basic vital signs in Helicobacter pylori positive and negative persons. Eur J Epidemiol. 2007;22(1):67-75.

19. Vo HD, Goli S, Gill R, Anderson V, Stefanov DG, Xu J, et al. Inverse correlation between Helicobacter pylori colonization and obesity in a cohort of inner city children. Helicobacter. 2015;20(1):64-8.

20. Lender N, Talley NJ, Enck P, Haag S, Zipfel S, Morrison M, et al. Review article: associations between helicobacter pylori and obesity: an ecological study. Aliment Pharmacol Ther. 2014;40(1):24-31.

21. Wu MS, Lee WJ, Wang HH, Huang SP, Lin JD. A case-control study of association of Helicobacter pylori infection with morbid obesity in Taiwan. Arch Intern Med. 2005;165(13):1552-5.

22. Rodriguez MMR, Araguez C, Pareja C, Alcaide J, Fernandez C, Garach M, et al. Carbohydrate metabolismo improvement after Helicobacter pylori eradication. Diabetes Metab. 2016;42(2):130-4.

23. Janjetic MA, Mantero P, Rua EC, Balcarce N, Palma GZ, Cataeleno M, et al. Dietary and anthropometric indicators of nutritional status in relation to Helicobacter pylori infection in a pediatric population. Br J Nutr. 2015;113(7):1113-9.

24. Oliveira TR, Sampaio HAC, Rocha DC, Lima GP, Vergara CMAC, Lima JWO, et al. Influência da infecção por Helicobacter pylori e de sua erradicação sobre o consumo e estado nutricional. Acta Gastroenterol Latinoam. 2015;45(4):288-94.

25. Coelho SB. Efeitos do amendoim sobre o apetite e níveis de leptina, insulina e ghrelina e análise de polimorfismos para o promotor da leptina e seu receptor, em indivíduos eutróficos [tese]. Viçosa: Universidade Federal de Viçosa; 2007.

26. Blundell J, De Graaf C, Hulshof T, Jebb S, Livingstone B, Lluch A. et al. Appetite control: methodological aspects of the evaluation of foods. Obes Rev. 2010;11(3):251-70.

27. Klok MD, Jakobsdottir S, Drent ML. The role of leptin and ghrelin in the regulation of food intake and body weight in humans: a review. Obes Rev. 2007;8(1):21-34.

\section{Endereço do primeiro autor:}

Laís Marinho Aguiar

Rua Paulo Moraes, 580/301

Bairro: Papicu

CEP: 60175-175 - Fortaleza - CE - Brasil

E-mail: laismarinhoaguiar@gmail.com.

\section{Endereço para correspondência:}

Helena Alves de Carvalho Sampaio

Rua Joaquim Nabuco, 500/402

Bairro: Meireles

CEP: 60125-120 - Fortaleza - CE - Brasil

E-mail: dr.hard2@gmail.com 